\title{
ON THE ERRORS OF THE EXPERIMENTAL POLE FIGURES
}

\author{
V. LUZIN and D. NIKOLAYEV \\ Joint Institute for Nuclear Research, Laboratory of Neutron Physics, 141980 \\ Dubna, Moscow region, Russia
}

(Received 20 June 1995)

\begin{abstract}
The present work is devoted to the extraction of pole figures from time-of-flight neutron spectra with different methods. Two aspects are mainly considered. The first one is to give a quantitative estimate of the errors in the extracted pole figures and to relate the errors of spectra to the errors of the pole figures. The second aspect is to elaborate criteria to determine the minimal exposure time, which is necessary to accumulate a spectrum with an acceptable error level.
\end{abstract}

KEY WORDS: Statistical error integral intensity profile fitting standard deviation pole figure.

\section{INTRODUCTION}

PFs, to reproduce ODF, are usually obtained from X-ray or neutron diffraction measurements, which are affected by experimental and instrumental errors. In the present work we introduce the concept of a quality of PFs and compare the quality of PFs, which are been extracted from time-of-flight (TOF) neutron spectra with two different methods.

The consideration of errors introduced by the experimental data and by the method of PF extraction was almost beyond the previous studies although that question should be of interest to obtain proper results and for further developments of experimental techniques or computation methods. We had found only one example in which measurement errors of PFs were obtained (Wenk, Kern, Pannetier, Höfler, Schäfer, Will and Brokmeier, 1988).

\section{FUNDAMENTALS}

The data processing procedure to extract PFs should fulfill the following conditions. (i) It is desirable to extract as many PFs as possible. The instrumental resolution is the natural limitation here. (ii) The extracted PFs should be of the highest accuracy. (iii) The large amount of experimental data requires a reliable (robust) automated procedure. (iv) All information included in the experimental data should be used for subsequent data processing.

To extract the PFs one has to find integrated intensity of the associated Bragg reflection in a certain spectrum because the pole density $P_{\mathrm{h} i}(y)$ and integrated intensity $S^{*}\left(\mathbf{h}_{i}, \mathbf{y}\right)$ must be proportional to the scattering volume fraction $V\left(\mathbf{h}_{i}, \| \mathbf{y}\right)$ : 


$$
P_{\mathrm{hi}}(\mathbf{y}) \sim S^{*}\left(\mathbf{h}_{i}, \mathbf{y}\right) \sim \frac{V\left(\mathbf{h}_{i}, \| \mathbf{y}\right)}{V},
$$

where $V$ is a whole scattering volume. Practically we have only an observation of Poisson counting statistics $I_{j}$ :

$$
I_{j}=\sum_{k=1}^{n} Y_{k j}
$$

where $n$ is the number of reactor impulses, $j$ is a channel and $\left\{Y_{j}\right\}_{k}$ is sample of Poisson value $Y_{j}=\{0,1,2, \ldots$.$\} with the parameter \lambda_{j}$ which is assumed to be $\lambda_{j}={ }_{n}^{1} \sum_{j} I_{j}$.

The spectrum $I_{j}$ may be considered as a linear mixture of peak and background intensity, which is superposed by statistical errors. This can be expressed as follows:

$$
I_{j}=\sum_{k=1} F_{k j}+B_{j}+Q_{j},
$$

where $j$ is a channel, $I_{j}$ is the spectrum to be analysed, $F_{k j}$ is $k$-th peak in the spectrum, $B_{j}$ is the background and $Q_{j}$ is statistical error.

Let's introduce the estimate of integrated intensity $S=S\left(\mathbf{h}_{i}, \mathbf{y}\right)=S\left(\mathbf{h}_{i}, \mathbf{y},\left\{I_{j}\right\}\right)$ as the area for the peak $\mathbf{h}_{i}$ in the corresponding TOF-spectrum for the direction $\mathbf{y}$. It may be determined by simple integration or by an iterative procedure. It is common to consider as a PF a normalized function of $S\left(\mathbf{h}_{i}, \mathbf{y}\right)$.

There are two different possibilities to obtain $S$, we named them parametric approach and nonparametric approach respectively. The parametric approach assumes a parametric model of the diffraction peak, which not identical with a parametric model of Poisson countings in each channel, which is adopted the same in the both cases.

The integrated peak intensity and the corresponding pole density are determined by numerical integration in a manually defined region of the spectrum. The background values determined beside the peak region. The integration is carried out with the NewtonCotes formulas of same order. In the simplest case of integration one obtains (Windsor, 1981):

$$
\begin{aligned}
& S=\sum_{j=j 1}^{2}\left(I_{j}-B\right), \\
& B=\left(\sum_{j=j 0}^{j 1-1} I_{j}+\sum_{j=j 2-1}^{j 3} I_{j}\right) /\left(j_{3}-j_{2}+j_{1}-j_{0}\right), \\
& \Delta S^{2}=\sum_{j=j 1}^{j 2}\left(I_{j}+B\right),
\end{aligned}
$$

where $\Delta S^{2}$ is the variance of the integrated intensity $S, B$ is background and $I_{j}$ corresponds to the intensity in channel $j, j_{l}, l=0,1,2,3$, are the limits of integration over the selected region. Here the Poisson counting statistics in each channel has been assumed.

This way to find out the integrated peak intensity doesn't consider the peak shape and the smoothness of the peak and background functions. It is difficult to resolve narrow overlapped peaks in this approach. Another disadvantage is its insensitivity to outliers.

Parameterization stands for application of the standard technique of profile fitting, which is well-known from literature (Albinati and Willis, 1982; Will, Parrish and Huang, 1983; Jansen, Schäfer and Will, 1988; Will, Schäfer and Merz, 1989). In contrast to 
the nonparametric approach the parametric one includes additional information about the peak shape, which was derived theoretically and also was observed experimentally.

By accumulating experience about the peak shape of TOF-spectra, the following one has been accepted for the NSHR-instrument in Dubna (Windsor, 1981; Albinati and Willis, 1982):

$$
\begin{aligned}
& \text { (i) } I(t)=H_{0} \exp \left\{-\frac{\left(t-t_{0}\right)^{2}}{2 \sigma_{1}^{2}}\right\}, t \leq t_{0} ; \\
& \text { (ii) } I(t)=H_{0} \exp \left\{-\frac{\left(t-t_{0}\right)^{2}}{2 \sigma_{2}^{2}}\right\}, \quad t_{0} \leq t \leq t_{1} ; \\
& \text { (iii) } I(t)=H_{0} \exp \left\{\frac{\left(\gamma^{2} \sigma_{2}^{2}\right)}{2 \sigma_{1}^{2}}\right\} \exp \left\{-\gamma\left(t-t_{0}\right)\right\}, \quad t \geq t_{1} \text {; }
\end{aligned}
$$

where $\sigma_{1}$ and $\sigma_{2}$ are determining the width of the Gauss regions at the left and right side of the peak maximum $H_{0}$, respectively, and $\gamma$ is the decay constant of the exponential region. The position $t_{1}=\mathrm{t}_{0}+\gamma \sigma_{2}^{2}$ ensures a smooth join between the regions. By means of this parameterization, each peak is described by at least five parameters and up to four background components (polynomial background). To find the bestfit curve the following minimization problem has to be solved:

$$
M=R^{T} W R \rightarrow \min ,
$$

where $R$ is the vector of the residuals: $R=I-C(p)$. The quantity $I$ is a one-column matrix with $n$ components $I_{j}$; the quantity $C(P)$ is a one-column matrix with $n$ components $C_{j}$, too. It approximates the observed quantity $I$ and is calculated from the set of parameters described by one-column matrix of parameters $P$ with $m<n$ components $P_{i}$. The weight matrix $W$ is proportional to the reciprocal variances $1 / D_{j}^{2}$ of the observed quantity $I_{j}$.

The minimization problem (3) is essentially nonlinear. This nonlinear least-squares problem may be reduced to a linear but iterative one by applying Taylor-series expansion. This deconvolution leads to a system of normal equations:

$$
\left(A^{T} W A\right) X=A^{T} W R,
$$

where $A$ is the design matrix of the least-squares problem:

$$
A_{j i}=\frac{\delta C_{j}}{\delta P_{i}}, \quad j=1, \ldots, n, \quad i=1, \ldots, m .
$$

and $X$ is the adjustment vector which provides new and better values of the parameters $\bar{P}$ after each iteration step: $P \rightarrow \bar{P}=P+X$. Solving (4) by performing LU-decomposition (Press, W. H. et al., 1992) we obtain the adjustment vector $X$, the new vector $\bar{P}=$ $P+X$ and $\bar{R}=I-C(\bar{P})$ as well. If convergence is achieved, one obtains the covariance matrix for the parameter vector:

$$
\Delta P^{2}=\frac{R^{T} W R}{n-m} N^{-1},
$$


If the vector of parameters $P$ has been determined, the integral of interest and its variance or standard deviation may be calculated easily. Such integrals for different directions $\vec{y}$ compose the PF. Corresponding standard deviations for different directions $\vec{y}$ also may be represented like PFs by SDPF (Standard Deviations of Pole Figures). In a similar manner, the PF and SDPF may be derived for the nonparametric method from formula (1). It is obvious, that a SDPF is not equivalent to a pole density distribution, but characterizes the spatial error distribution.

\section{APPLICATION: AN EXAMPLE}

TOF-spectra of a monophase quartz rock sample (cylinder of $20 \mathrm{~mm}$ diameter and 20 $\mathrm{mm}$ height) were measured repeatedly with exposure times 2,4 and 8 minutes at NSHR (Neutron Spectrometer of High Resolution) at the fast pulsed reactor IBR-2 at the Joint Institute for Nuclear Research, Dubna (Feldmann, Betzl, Kleinsteuber and Walther, 1991 and Helming, Voitus and Walther, 1992). From this independent measurement all exposure times in the range from 2 minutes up to 14 may be composed in steps of 2 minutes. For illustration we consider the experimental PFs extracted by both methods of the crystallographic directions $\{01 \overline{1} 1\} /\{10 \overline{1} 1\}$ and $\{11 \overline{2} 0\}$ (fig. 2.a.)

To describe the discrepancy between experimental PF and "true" (reference) PF $R P_{i}$ (ع) values may be used (Matthies, Wenk and Vinel, 1988):

$$
R P_{i}(\varepsilon)=\sum_{j=1}^{J} R P_{i, j} / \sum_{j=1}^{J} \Theta\left[\varepsilon, P_{\mathrm{h} i}\left(\mathrm{y}_{j}\right)\right] ; \quad j=1, \ldots, J, \quad i=1, \ldots, I
$$

where

$$
R P_{i, i}(\varepsilon)=\Theta\left[\varepsilon, P_{\mathrm{h} i}\left(\mathrm{y}_{j}\right)\right] \frac{\Delta P_{\mathrm{h} i}\left(\mathrm{y}_{j}\right)}{P_{\mathrm{hi}}\left(\mathrm{y}_{j}\right)}
$$

and the "difference pole figure" is given by

$$
\Delta P_{\mathrm{h} i}\left(\mathrm{y}_{j}\right)=P_{\mathrm{h} i}\left(\mathrm{y}_{j}\right)-P_{\mathrm{h} i}^{*}\left(\mathrm{y}_{j}\right)
$$

Here $P_{\mathrm{h} i}^{*}\left(\mathrm{y}_{j}\right)$ denotes the "true" (reference) PF and $\Theta(\varepsilon, x)$ is a selecting function

$$
\Theta[\varepsilon, x]=\left\{\begin{array}{l}
0, x \leq \varepsilon \\
1, x>\varepsilon
\end{array}\right.
$$

In analogy, we introduce the $Q P F_{i}(\varepsilon)$ value (Quality of Pole Figure) for the pole figures $P_{\mathrm{h} i}\left(\mathrm{y}_{i}\right)$

$$
Q P F_{i}(\varepsilon)=\sum_{j=1}^{J} Q P F_{i, j} / \sum_{j=1}^{J} \Theta\left[\varepsilon, P_{\mathrm{h} i}\left(\mathrm{y}_{j}\right)\right]
$$

where

$$
Q P F_{i, j}(\varepsilon)=\Theta\left[\varepsilon, P_{\mathrm{h} i}\left(\mathrm{y}_{j}\right)\right], \frac{S D P F_{\mathrm{h} i}\left(\mathrm{y}_{j}\right)}{P_{\mathrm{h} i}\left(\mathrm{y}_{j}\right)}
$$


$\{01 \overline{1} 1\} /\{10 \overline{1} 1\}$
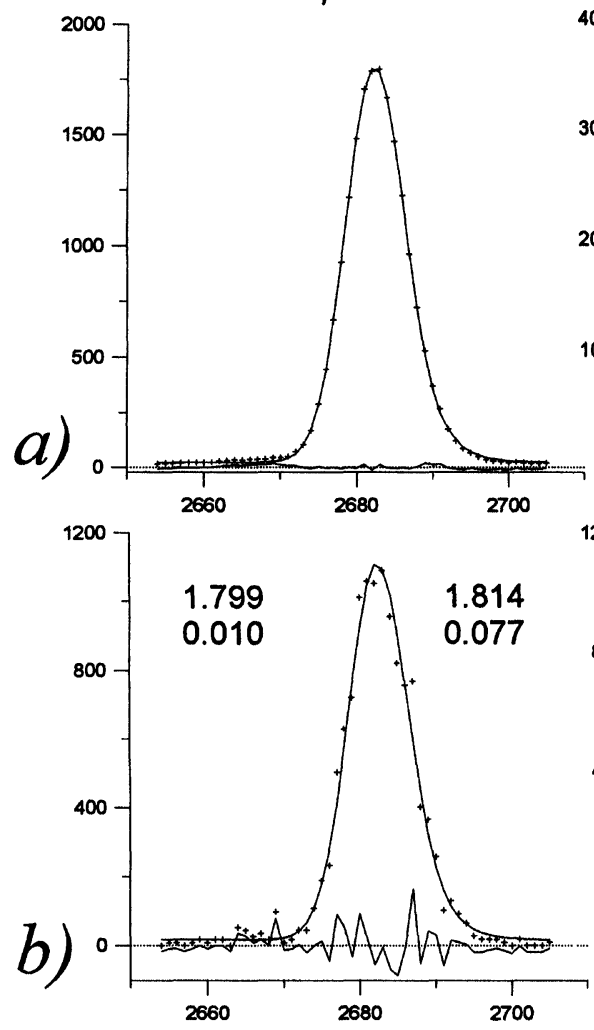

$\{11 \overline{2} 0\}$
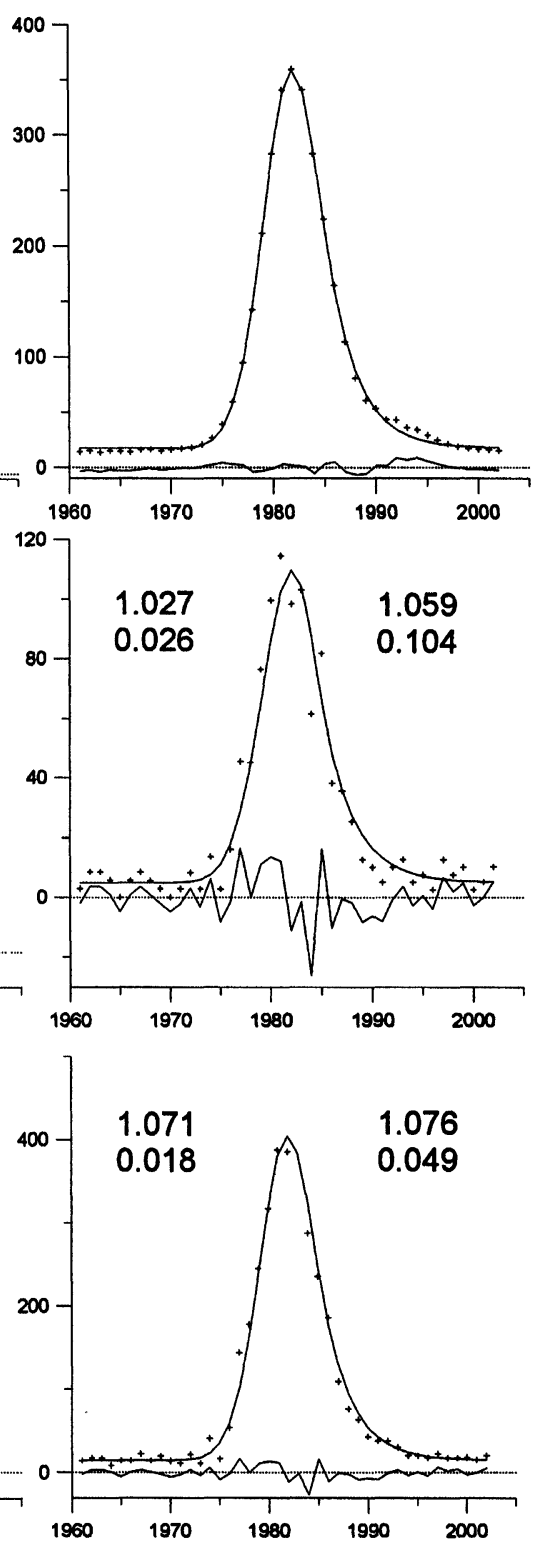

Figure 1 a) Patterns of TOF-spectra after summation of 102 individual spectra to show the peak shape. Patterns of TOF-spectra for exposure times b) $4 \mathrm{~min}$ and c) $14 \mathrm{~min}$ to illustrate the differences in counting statistics. Measured (crosses) and calculated (lines) diffraction peaks are plotted. At the bottom of peak the differences between measured and calculated values are plotted. At the left of the peak the corresponding PF value and SDPF value derived by profile analysis are given. At the right of the peak these values are given for the nonparametric methods. Miller indices are indicated at the top of the figure. 


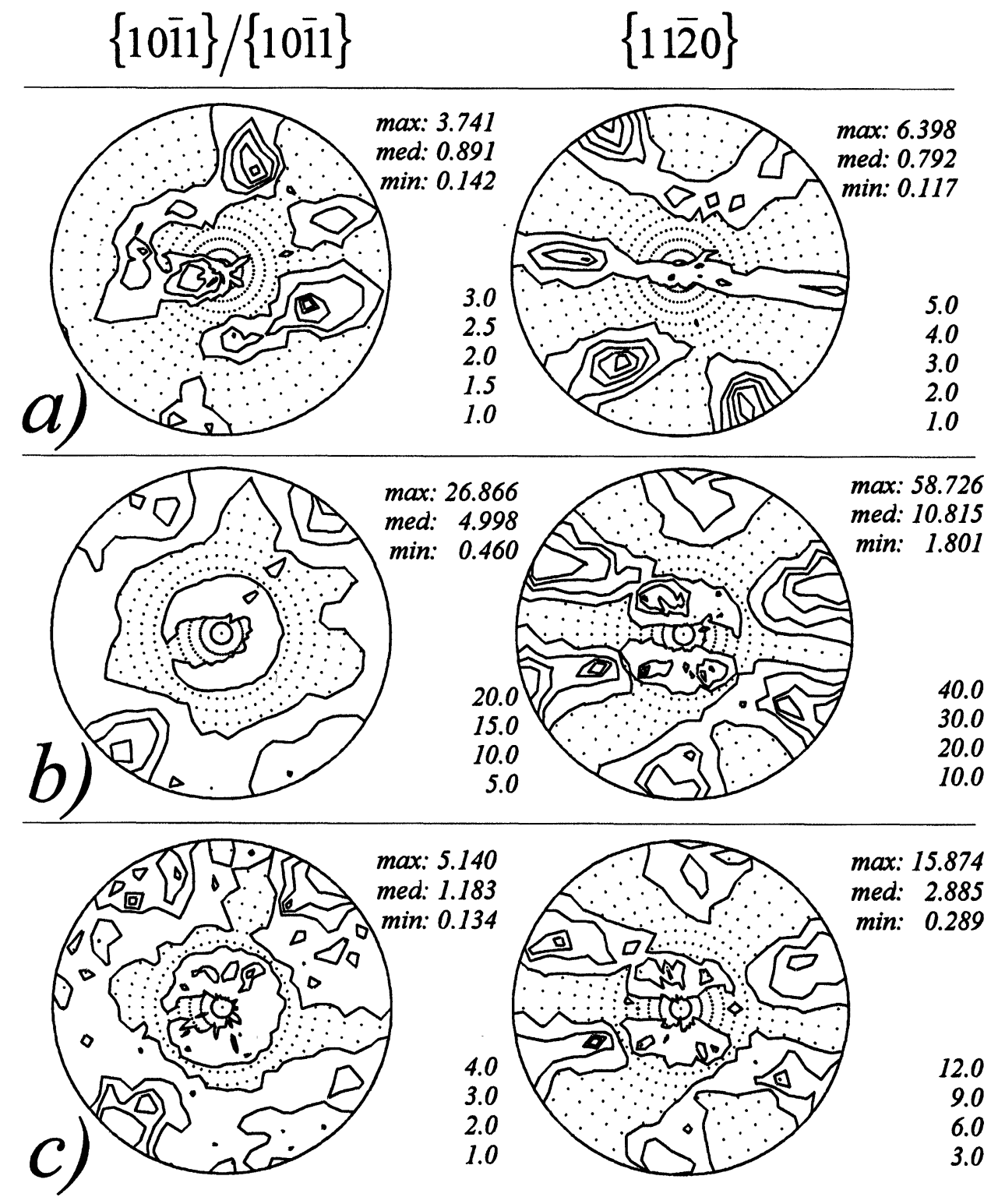

Figure 2 a) Experimental PFs $\{01 \overline{1} 1\} /\{10 \overline{1} 1\}$ and $\{1 \overline{1} \overline{0} 0\}$ for exposure time of $14 \mathrm{~min}$. The functions $S D P F_{\mathrm{h} i}\left(\mathrm{y}_{j}\right) / P F_{\mathrm{h} i}\left(\mathrm{y}_{j}\right)$ for $\left.\mathrm{b}\right)$ the nonparametric method and c) the parametric method are given for exposure time of 4 min. 

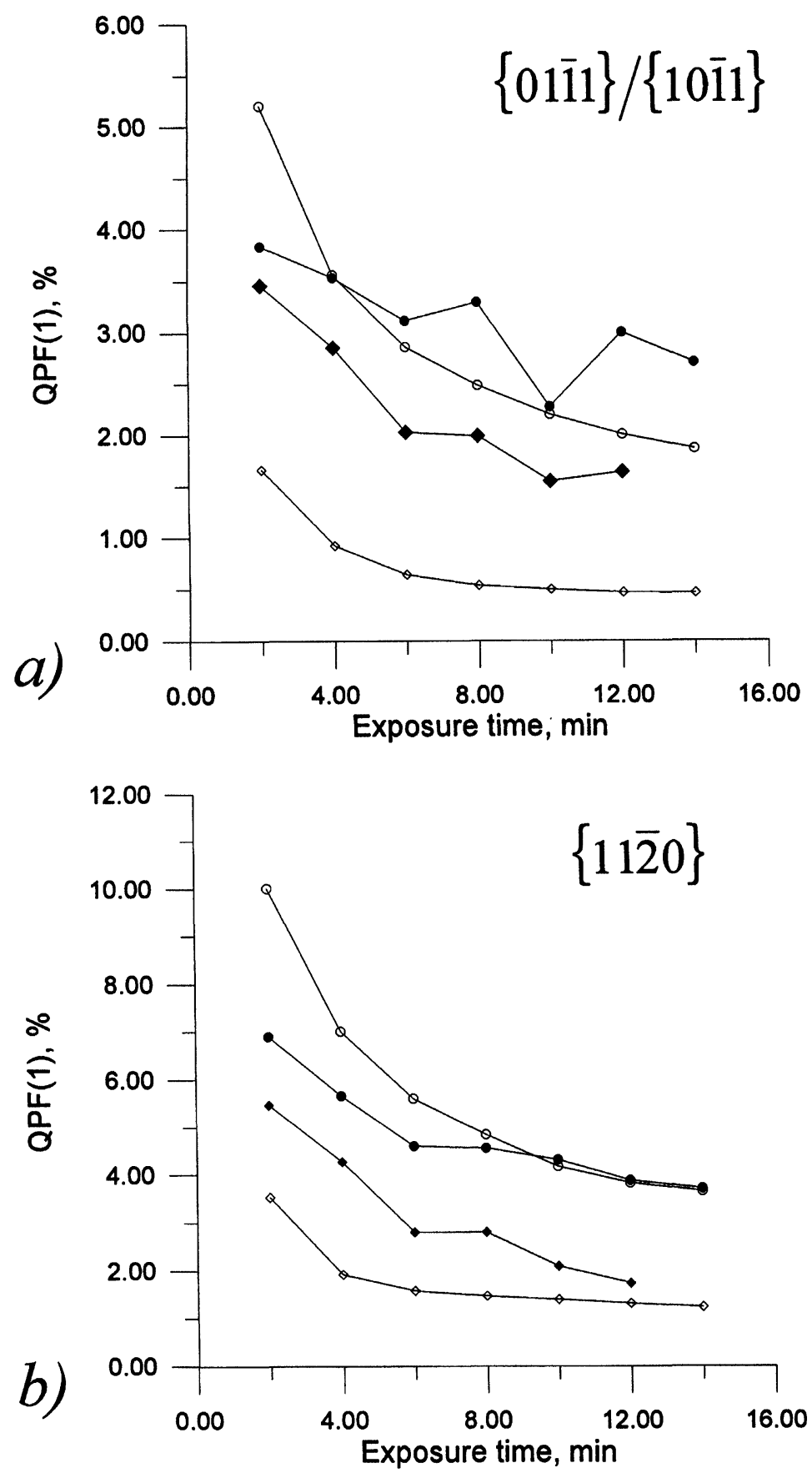

Figure 3 Dependences of $Q P F_{i}(1)$ values $(\bigcirc-$ the nonparametric method, $\diamond-$ the LSM-method) on exposure time for a) $\{0111\} /\{1011\}$, and b) $\{1120\}$. By shaded sings $(\bullet$ nonparametric method, $\diamond$ - LSM-method) the "real" errors of PFs or $R p_{i}(1)$ values in dependence on exposure time are indicated. 
The $Q P F_{i}(\varepsilon)$ values represent a numerical criterion for the quality of the experimental PF. In contrast to the $R P_{i}(\varepsilon)$ values they describe the estimates of deviations of an experimental PF from a "true" $P F$.

The dependences of $Q P F_{i}(\varepsilon)$ values on exposure time are shown in Fig. 3 for $\{01 \overline{1}$ $1\} /\{10 \overline{1} 1\}$ and $\{11 \overline{2} 0\}$ for both data processing methods and for $\varepsilon=1$. Corresponding points are labelled by unshaded circles and squares. Shaded circles and squares indicate $R P_{i}(\varepsilon)$ values (or the "real" errors of PFs) where the "true" PFs were evaluated from the PFs obtained from 14-minutes spectra by the LSM-method.

One can clearly see that the LSM-method gives a lower boundary of error evaluation. The limit into what the LSM-curve of quality pass $(\sim 0.5 \%$ for $\{01 \overline{1} 1\} /\{10 \overline{1} 1\}$ and $\sim 1 \%$ for $\{11 \overline{2} 0\})$ is due to a gap between peak model and real peak shape and due to other reason except that of statistic (a limited precision of numbers, numerical error of calculations).

The dependences analogous to the presented can be derived for all required PFs (peaks) and for any material. So to evaluate a minimal exposure time for a predetermined set of PFs and predetermined accuracy it is necessary to evaluate this time only for the lowest intensity PF (or diffraction peak) from corresponding $Q P F_{i}(\varepsilon)$ dependences.

\section{CONCLUSION}

To characterize quantitatively the errors of an experimental PF the functions $\operatorname{SDPF}_{\mathrm{h} i}\left(\mathrm{y}_{j}\right)$ and the value $Q P F_{i}(\varepsilon)$ were proposed. The $S D P F_{\mathrm{h} i}\left(\mathrm{y}_{j}\right)$ contains an information on the special distribution of errors. The $Q P F_{i}(\varepsilon)$ values are integral errors of PFs and are interpreted to represent quantitative characteristics of a quality of PFs.

From the two considered approaches of PF extraction from TOP-spectra the parametric approach (profile analysis) leads to PFs of better quality. It is concluded, that a minimal possible error for each particular PF was achieved almost for the exposure time about 4-8 minutes. So, for the NSHR-instrument the most reasonable exposure time is 6 minutes (only for the considered sample or comparable ones).

The $Q P F_{i}(\varepsilon)$ value in contradiction to the $R P_{i}(\varepsilon)$ value doesn't require the knowledge of "true" $\mathrm{PF}$, which is generally unknown, it may be derived only from the spectra. The $Q P F_{i}(\varepsilon)$ value can be a quantitative criterion to obtain some impression about the accuracy of ODF reproduction. Such an impression cannot be obtained from $R P_{i}(\varepsilon)$ values.

\section{References}

Albinati, A. and Willis, T. M. (1982). J. Appl. Cryst. 15, 361-374.

Feldmann, K., Betzl, M., Kleinsteuber, W. and Walther, K. (1991). Textures Microstruct. 14-18, 59-64.

Jansen, E., Schäfer, W. and Will, G. (1988). J. Appl. Cryst. 21, 228-239.

Hampel, F. R., Ronchetti, E. M., Rousseeuw, P. J. and Stahel, W. A. (1986). Robust Statistics. The Approach Based on Influence Functions. N. -Y.: John Wiley and Sons.

Helming, K., Voitus, W. and Walther, K. (1992). Physica B. 180 \& 181, 1025-1028.

Huber, P. J. (1981). Robust Statistics. N. -Y.: John Wiley and Sons.

Matthies, S., Wenk, H. -R. and Vinel, G. W. (1988). J. Appl. Cryst. 21, 285-304.

Press, W. J. et al. (1992). Numerical recires in $C:$ the art of scientific computing. Combridge University Press.

Wenk, H. -R., Kern, H., Pannetier, J., Höfler, S., Schäfer, W., Will, G. and Brokmeier, H. -G. (1991). Proceedings of Eighth International Conference on Textures of Materials - ICOTOM8, 229-234.

Will, G., Parrish, W. and Huang, T. C. (1983). J. Appl. Cryst. 16, 611-622.

Will, G., Schafer, W. and Merz, P. (1989). Textures Microstruct. 10, 375-387.

Windsor, C. G. (1981). Pulsed Neutron Scattering. London: Taylor and Francis. 